Pacific Journal of Mathematics

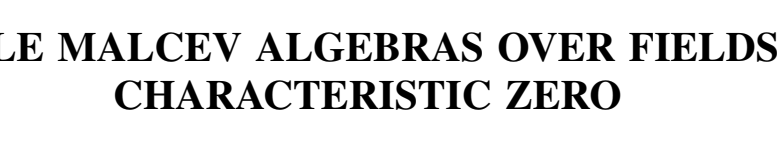




\title{
SIMPLE MALCEV ALGEBRAS OVER FIELDS OF CHARACTERISTIC ZERO
}

\author{
Arthur A. SAgLE
}

1. Introduction. Malcev algebras are a natural generalization of Lie algebras suggested by introducing the commutator of two elements as a new multiplicative operation in an alternative algebra [3]. The defining identities obtained in this way for a Malcev algebra $A$ are

$$
\begin{gathered}
x y=-y x \\
x y \cdot x z=(x y \cdot z) x+(y z \cdot x) x+(z x \cdot x) y
\end{gathered}
$$

for all $x, y, z \in A$. Since Albert [1] has shown that every simple alternative ring which contains an idempotent not its unity quantity is either associative or the split Cayley-Dickson algebra $C$, it is natural to see if a simple Malcev algebra can be obtained from $C$. In [3] a seven dimensional simple non-Lie Malcev algebra $A^{*}$ is obtained from $C$ and is discussed in detail. In this paper we shall prove the following

Theorem. Let $A$ be a finite dimensional simple non-Lie Malcev algebra over an algebraically closed field of characteristic zero. Furthermore assume $A$ contains an element $u$ such that the right multiplication by $u, R_{u}$, is not a nilpotent linear transformation. Then $A$ is isomorphic to $A^{*}$.

The necessary identities and notation from [3] for any algebra $A$ are repeated here for convenience:

$$
\begin{array}{ll}
\text { Commutator, } & (x, y)=[x, y]=x y-y x \\
\text { Associator, } & (x, y, z)=x y \cdot z-x \cdot y z \\
\text { Jacobian, } & J(x, y, z)=x y \cdot z+y z \cdot x+z x \cdot y
\end{array}
$$

for $x, y, z \in A$. If $h\left(x_{1}, \cdots, x_{n}\right)$ is a function of $n$ indeterminates such that for any $n$ subsets $B_{i}$ of $A$ and $b_{i} \in B_{i}$, the elements $h\left(b_{1}, \cdots, b_{n}\right)$ are in $A$, then $h\left(B_{1}, \cdots, B_{n}\right)$ will denote the linear subspace of $A$ spanned by all of the elements $h\left(b_{1}, \cdots, b_{n}\right)$.

For a Malcev algebra $A$ of characteristic not 2 or 3 , we shall use the following identities and theorems from [3]:

$$
J(x, y, x z)=J(x, y, z) x
$$

Received September 2, 1961. The author would like to thank Professor L. J. Paige for his assistance in the preparation of the manuscript. This research was sponsored in part by the National Science Foundation under NSF Grant G-9504. 


$$
\begin{aligned}
& J(x, y, w z)+J(w, y, x z)=J(x, y, z) w+J(w, y, z) x \\
& 2 w J(x, y, z)=J(w, x, y z)+J(w, y, z x)+J(w, z, x y) \\
& J(w x, y, z)=w J(x, y, z)+J(w, y, z) x-2 J(y z, w, x) \\
& x y \cdot z w=x(w y \cdot z)+w(y z \cdot x)+y(z x \cdot w)+z(x w \cdot y)
\end{aligned}
$$

for all $w, x, y, z \in A$. If $N=\{x \in A: J(x, A, A)=0\}$, then it is shown in [3] that $N$ is an ideal of $A$ which is a Lie subalgebra and furthermore for $a, b \in A$

$$
J(a, b, A)=0 \quad \text { implies } a b \in N .
$$

It is also shown in [3] that $J(A, A, A)$ is an ideal of $A$. Thus if $A$ is a simple non-Lie Malcev algebra we have

$$
N=0 \text { and } A=J(A, A, A) .
$$

We shall assume throughout this paper that $A$ is a finite dimensional simple non-Lie Malcev algebra over an algebraically closed field $F$ of characteristic not 2 or 3 containing an element $u$ such that $R_{u}$ is not a nilpotent linear tansformation. In $\S 2$ the basic multiplicative identities are derived using methods analogous to those of Lie algebras. Decomposing $A=A_{0} \oplus A_{\alpha} \oplus \cdots \oplus A_{\gamma}$ into weight spaces relative to $R_{u}$ [2; page 132] we prove the block multiplication identities $A_{\alpha} A_{\beta} \subset$ $A_{\alpha+\beta}$ if $\alpha \neq \beta, A_{\alpha}^{2} \subset A_{-\alpha}$ and $A_{0}^{2}=0$. Further identities are derived in $\S 3$ which lead to the important result that there exists a nonzero weight $\alpha$ such that $A=A_{0} \oplus A_{\alpha} \oplus A_{-\alpha}$ where $A_{0}=A_{\alpha} A_{-\alpha}$.

In $\S 4$ we show that $R\left(A_{0}\right)$, the set of right multiplications $R_{x_{0}}$ by elements $x_{0} \in A_{0}$, is a set of commuting linear transformations on the subspaces $A_{0}, A_{\alpha}$ and $A_{-\alpha}$. Analogous to Lie algebras we decompose $A=A_{0} \oplus A_{\alpha} \oplus A_{-\alpha}$ into weight spaces relative to $R\left(A_{0}\right)$ [2; page 133] and thus find a basis of $A$ which simultaneously triangulates the matrices of $R\left(A_{0}\right)$. We now introduce the trace form, $(x, y)=\operatorname{trace} R_{x} R_{y}$, in $\S 5$ and assume for the remainder of the paper that the algebraically closed field is of characteristic zero. With this and the results of $\S 4$ we easily show that $(x, y)$ is a nondegenerate invariant form on $A=$ $A_{0} \oplus A_{\alpha} \oplus A_{-\alpha}$ and $A_{0}=u F$.

In $\S 6$ we show that $R_{u}$ has a diagonal matrix of the form

$$
\left[\begin{array}{rrrr}
0 & & 0 \\
& \alpha I & \\
0 & & -\alpha I
\end{array}\right]
$$

Using this and a few more identities we show in $\S 7$ that the simple Malcev algebra $A=A_{0} \oplus A_{\alpha} \oplus A_{-a}$ is isomorphic to the seven dimen- 
sional algebra $A^{*}$.

2. Basic multiplication identities. Let $R_{u}(u \in A)$ be a fixed nonnilpotent linear transformation and decompose the simple Malcev algebra $A$ into the weight space direct sum $A=A_{0} \oplus A_{\alpha} \oplus \cdots \oplus A_{\gamma}$ relative to $R_{u}$ where the weight space of $R_{u}$,

$$
A_{a}=\left\{x \in A: x\left(a I-R_{u}\right)^{k}=0 \text { for some integer } k>0\right\},
$$

is a nonzero $R_{u}$-invariant subspace of $A$ corresponding to the weight $a$ of $R_{u}$. Let $x_{\alpha} \in A_{\alpha}, x_{\beta} \in A_{\beta}$, then using (1.6)

$$
J\left(u, x_{\alpha}, x_{\beta}\right) R_{u}=J\left(u, x_{\alpha}, x_{\beta}\right) u=J\left(u, x_{\alpha}, u x_{\beta}\right)=-J\left(u, x_{\alpha}, x_{\beta} R_{u}\right)
$$

and therefore

$$
J\left(u, x_{\alpha}, x_{\beta}\right)\left(\beta I+R_{u}\right)=J\left(u, x_{\alpha}, x_{\beta}\left(\beta I-R_{u}\right)\right) .
$$

Now letting $y_{\beta}=x_{\beta}\left(\beta I-R_{u}\right) \in A_{\beta}$ we have

$$
\begin{aligned}
J\left(u, x_{\alpha}, x_{\beta}\left(\beta I-R_{u}\right)^{2}\right) & =J\left(u, x_{\alpha}, y_{\beta}\left(\beta I-R_{u}\right)\right) \\
& =J\left(u, x_{\alpha}, y_{\beta}\right)\left(\beta I+R_{u}\right) \\
& =J\left(u, x_{\alpha}, x_{\beta}\left(\beta I-R_{u}\right)\right)\left(\beta I+R_{u}\right) \\
& =\left(u, x_{\alpha}, x_{\beta}\right)\left(\beta I+R_{u}\right)^{2} .
\end{aligned}
$$

Continuing by induction we obtain

$$
J\left(u, x_{\alpha}, x_{\beta}\right)\left(\beta I+R_{u}\right)^{n}=J\left(u, x_{\alpha}, x_{\beta}\left(\beta I-R_{u}\right)^{n}\right)
$$

for every integer $n$. Since $x_{\beta} \in A_{\beta}$ there exists an integer $N$ such that $0=J\left(u, x_{\alpha}, x_{\beta}\left(\beta I-R_{u}\right)^{N}\right)=J\left(u, x_{\alpha}, x_{\beta}\right)\left(\beta I+R_{u}\right)^{N}$ and this shows $J\left(u, x_{\alpha}, x_{\beta}\right) \in A_{-\beta}$. Now interchanging the roles of $x_{\beta}$ and $x_{\alpha}$ in (2.1) we also obtain $J\left(u, x_{\alpha}, x_{\beta}\right) \in A_{-\alpha}$ and thus

$$
J\left(u, A_{\alpha}, A_{\beta}\right) \subset A_{-\alpha} \cap A_{-\beta} .
$$

From (2.2) we have the following relations

$$
\begin{gathered}
J\left(u, A_{\alpha}, A_{\alpha}\right) \subset A_{-\alpha} \\
J\left(u, A_{\alpha}, A_{\beta}\right)=0 \text { if } \alpha \neq \beta .
\end{gathered}
$$

We shall now prove

$$
A_{\alpha} A_{\beta} \subset A_{\alpha+\beta} \text { if } \alpha \neq \beta \text {. }
$$

For if $\alpha \neq \beta$ and $x_{\alpha} \in A_{\alpha}, x_{\beta} \in A_{\beta}$ we have by (2.4),

$$
0=J\left(u, x_{\alpha}, x_{\beta}\right)=\left(x_{\alpha} x_{\beta}\right) R u-x_{\alpha} R_{u} \cdot x_{\beta}-x_{\alpha} \cdot x_{\beta} R_{u} ;
$$

that is, $\left(x_{\alpha} x_{\beta}\right) R_{u}=x_{\alpha} R_{u} \cdot x_{\beta}+x_{\alpha} \cdot x_{\beta} R_{u}$ and so $R_{u}$ is a derivation of 
$A_{\alpha} A_{\beta}$ into $A_{\alpha} A_{\beta}$. This yields

$$
\left(x_{\alpha} x_{\beta}\right)\left(R_{u}-(\alpha+\beta) I\right)=x_{\alpha}\left(R_{u}-\alpha I\right) \cdot x_{\beta}+x_{\alpha} \cdot x_{\beta}\left(R_{u}-\beta I\right)
$$

and in the usual was we prove the Lebnitz rule for derivations which then yields that for some integer $N,\left(x_{\alpha} x_{\beta}\right)\left(R_{u}-(\alpha+\beta) I\right)^{N}=0$ and therefore $x_{\alpha} x_{\beta} \in A_{\alpha+\beta}$. In particular we have

$$
A_{0} A_{\alpha} \subset A_{\alpha} \text { if } \alpha \neq 0 \text {. }
$$

We shall now investigate $A_{0}$ more closely. Let $x_{\alpha} \in A_{\alpha}, x_{\beta} \in A_{\beta}$ and $x_{0} \in A_{0}$, then by (1.7) $J\left(x_{0}, x_{\beta}, u x_{\alpha}\right)+J\left(u, x_{\beta}, x_{0} x_{\alpha}\right)=J\left(x_{0}, x_{\beta}, x_{\alpha}\right) u+$ $J\left(u, x_{\beta}, x_{\alpha}\right) x_{0}$. Therefore if $0 \neq \alpha \neq \beta$ we have by (2.4) $J\left(x_{0}, x_{\beta}, u x_{\alpha}\right)=$ $J\left(x_{0}, x_{\beta}, x_{\alpha}\right) u$. This yields $J\left(x_{0}, x_{\beta}, x_{\alpha}\left(\alpha I-R_{u}\right)\right)=J\left(x_{0}, x_{\beta}, x_{\alpha}\right)\left(\alpha I+R_{u}\right)$ and as in the proof of (2.4) we obtain

$$
J\left(A_{0}, A_{\alpha}, A_{\beta}\right)=0 \text { if } 0 \neq \alpha \neq \beta \neq 0 .
$$

Next let $x_{0}, y_{0} \in A_{0}$ and $x_{\alpha} \in A_{\alpha}$ where $\alpha \neq 0$, then using (1.9), (2.4) and (2.6) we have

$$
\begin{aligned}
J\left(x_{0} u, y_{0}, x_{\alpha}\right) & =x_{0} J\left(u, y_{0}, x_{\alpha}\right)+J\left(x_{0}, y_{0}, x_{\alpha}\right) u-2 J\left(y_{0} x_{\alpha}, x_{0}, u\right) \\
& =J\left(x_{0}, y_{0}, x_{\alpha}\right) u
\end{aligned}
$$

and in general we have $J\left(x_{0} R_{u}^{n}, y_{0}, x_{\alpha}\right)=J\left(x_{0}, y_{0}, x_{\alpha}\right) R_{u}^{n}$ which implies $J\left(x_{0}, y_{0}, x_{\alpha}\right) \in A_{0}$. Now by (1.7), $J\left(x_{0}, y_{0}, u x_{\alpha}\right)+J\left(u, y_{0}, x_{0} x_{\alpha}\right)=$ $J\left(x_{0}, y_{0}, x_{\alpha}\right) u+J\left(u, y_{0}, x_{\alpha}\right) x_{0}$; and using (2.4) and (2.6) we obtain $J\left(x_{0}, y_{0}, x_{\alpha} R_{u}\right)=-J\left(x_{0}, y_{0}, x_{\alpha}\right) R_{u}$ which implies $J\left(x_{0}, y_{0}, x_{\alpha}\left(R_{u}-\alpha I\right)\right)=$ $-J\left(x_{0}, y_{0}, x_{\alpha}\right)\left(R_{u}+\alpha I\right)$. Thus, as usual, we have $J\left(x_{0}, y_{0}, x_{\alpha}\right) \in A_{-a}$ and therefore $J\left(x_{0}, y_{0}, x_{\alpha}\right) \in A_{0} \cap A_{-\alpha}$ which proves

$$
J\left(A_{0}, A_{0}, A_{\alpha}\right)=0 \text { if } \alpha \neq 0 .
$$

We shall now show $A_{0}^{2} \subset A_{0}$. From our basic decomposition $A=$ $A_{0} \oplus A_{\alpha} \oplus \cdots \oplus A_{\gamma}$ relative to $R_{u}$ we can find a basis $\left\{x_{1}(\tau), \cdots, x_{m}(\tau)\right\}$ ( $m=m_{\tau}$ ) of $A_{\tau}$ such that

$$
x_{i}(\tau) R_{u}=\sum_{j=1}^{i-1} a_{i j} x_{j}(\tau)+\tau x_{i}(\tau)
$$

where $\tau, a_{i j} \in F$ and $i=1, \cdots, m$. In particular let $\left\{x_{1}(0), \cdots, x_{n_{0}}(0)\right\} \equiv$ $\left\{x_{1}, \cdots, x_{n}\right\}$ be the above type for $A_{0}$. Then $x_{1} R_{u}=0$ and

$$
x_{i} R_{u}=\sum_{k=1}^{i-1} a_{i k} x_{k} \quad(i=2, \cdots, n) .
$$

Furthermore,

$$
\begin{aligned}
J\left(u, x_{i}, x_{j}\right) & =\left(x_{i} x_{j}\right) R_{u}+x_{j} R_{u} \cdot x_{i}+x_{j} \cdot x_{i} R_{u} \\
& =\left(x_{i} x_{j}\right) R_{u}+\sum_{k=1}^{j-1} a_{j k} x_{k} x_{i}+\sum_{k=1}^{i-1} \alpha_{i k} x_{j} x_{k}
\end{aligned}
$$


with the understanding that $a_{10}=0$.

Using (1.6) and operating on both sides of the previous equation with $R_{u}^{n}$, we obtain

$$
\begin{aligned}
(-1)^{n} J\left(u, x_{i}, x_{j} R_{u}^{n}\right)= & J\left(u, x_{i}, x_{j}\right) R_{u}^{n} \\
= & \left(x_{i} x_{j}\right) R_{u}^{n+1}+\sum_{k=1}^{j-1} a_{j k}\left(x_{k} x_{i}\right) R_{u}^{n} \\
& +\sum_{k=1}^{i-1} a_{i k}\left(x_{j} x_{k}\right) R_{u}^{n} .
\end{aligned}
$$

Now by assuming $i<j$ and choosing $n$ large enough, a simple inductive argument yields $x_{i} x_{j} \in A_{0}$ for all $i$ and $j$. Thus $A_{0}^{2} \subset A_{0}$.

Using (1.8), $A_{0}^{2} \subset A_{0}$ and (2.8) we have

$$
A_{\alpha} J\left(A_{0}, A_{0}, A_{0}\right) \subset J\left(A_{\alpha}, A_{0}, A_{0}^{2}\right) \subset J\left(A_{\alpha}, A_{0}, A_{0}\right)=0 \text { for } \alpha \neq 0 \text {. }
$$

Thus, $A J\left(A_{0}, A_{0}, A_{0}\right) \subset \sum_{\alpha} A_{\alpha} J\left(A_{0}, A_{0}, A_{0}\right)=A_{0} J\left(A_{0}, A_{0}, A_{0}\right) \subset J\left(A_{0}, A_{0}, A_{0}\right)$, or $J\left(A_{0}, A_{0}, A_{0}\right)$ is an ideal of $A$. But since $J\left(A_{0}, A_{0}, A_{0}\right) \subset A_{0} \neq A$ and $A$ is simple we have

$$
J\left(A_{0}, A_{0}, A_{0}\right)=0 \text {. }
$$

Now using (2.8) and (2.10) we have $J\left(A_{0}, A_{0}, A\right)=\sum_{\alpha} J\left(A_{0}, A_{0}, A_{a}\right)=$ 0 and by (1.11) and (1.12),

$$
A_{0}^{2} \subset N=0 \text {. }
$$

In particular this means the kernel of $R_{u}$ is $A_{0}$.

We shall now show $A_{\alpha}^{2} \subset A_{-\alpha}$. Let $x_{\alpha}, y_{\alpha} \in A_{\alpha}$ for $\alpha \neq 0$, then by (2.3) $J\left(u, x_{\alpha}, y_{\alpha}\right)=\left(x_{\alpha} y_{\alpha}\right) R_{u}+y_{\alpha} R_{u} \cdot x_{\alpha}+y_{\alpha} \cdot x_{\alpha} R_{u}=w_{-\alpha} \in A_{-\alpha}$. Therefore $\left(x_{\alpha} y_{\alpha}\right) R_{u}=x_{\alpha} R_{u} \cdot y_{\alpha}+y_{\alpha} \cdot y_{\alpha} R_{u}+w_{-\alpha}$ which yields

$$
\left(x_{\alpha} y_{\alpha}\right)\left(R_{u}-2 \alpha I\right)=x_{\alpha}\left(R_{u}-\alpha I\right) \cdot y_{\alpha}+x_{\alpha} \cdot y_{\alpha}\left(R_{u}-\alpha I\right)+w_{-\alpha}^{(1)} .
$$

By induction we obtain

$$
\left(x_{\alpha} y_{\alpha}\right)\left(R_{u}-2 \alpha I\right)^{n}=w_{-\alpha}^{(n)}+\sum_{r=0}^{n} C_{n, r} x_{\alpha}\left(R_{u}-\alpha I\right)^{n-r} \cdot y_{\alpha}\left(R_{u}-\alpha I\right)^{r}
$$

where $w_{-\alpha}^{(n)} \in A_{-\alpha}$. Therefore for large enough $N,\left(x_{\alpha} y_{\alpha}\right)\left(R_{u}-2 \alpha I\right)^{N} \in A_{-\alpha}$. Now let $x_{\alpha} y_{\alpha}=\sum_{\gamma} z_{\gamma}$ where $z_{\gamma} \in A_{\gamma}$, then $\left(x_{\alpha} y_{\alpha}\right)\left(R_{u}-2 \alpha I\right)^{N}=$ $\sum_{\gamma} z_{\gamma}\left(R_{u}-2 \alpha I\right)^{N} \in A_{-\alpha}$. Therefore by the $R_{u}$-invariance of the $A_{\gamma}$ and the uniqueness of the decomposition $A=A_{0} \oplus A_{\alpha} \oplus \cdots \oplus A_{\lambda}, z_{\gamma}\left(R_{u}\right.$ $2 \alpha I)^{N}=0$ if $\gamma \neq-\alpha$. Thus if $\gamma \neq-\alpha, z_{\gamma} \in A_{2 \alpha}$. Therefore $x_{\alpha} y_{\alpha}=$ $z_{2 \alpha}+z_{-\alpha}$ which proves

$$
A_{a}^{2} \subset A_{2 \alpha} \oplus A_{-a}
$$


LEMMA 2.13. $J\left(u, A_{\alpha}^{2}, A_{2 \alpha}\right)=0$.

Proof. Using (2.12), (2.7) and (2.3) we have

$$
J\left(u, A_{\alpha}^{2}, A_{2 \alpha}\right) \subset J\left(u, A_{-\alpha}, A_{2 \alpha}\right)+J\left(u, A_{2 \alpha}, A_{2 \alpha}\right) \subset J\left(u, A_{2 \alpha}, A_{2 \alpha}\right) \subset A_{-2 \alpha} .
$$

Now for any $x, y \in A_{\alpha}, z \in A_{2 \alpha}$ we have by (1.7) $J(z, u, x y)+J(x, u, z y)=$ $J(z, u, y) x+J(x, u, y) z$ and using (2.4), (2.5) and (2.3) this yields $J(z, u, x y)=J(x, u, y) z \in A_{-\alpha} \cdot A_{2 \alpha} \subset A_{\alpha}$. Combining these results we have $J\left(u, A_{\alpha}^{2}, A_{2 \alpha}\right) \subset A_{\alpha} \cap A_{-2 \alpha}=0$.

Now let $w \in A_{2 \alpha}, x, y \in A_{\alpha}$ and $x y=z_{2 \alpha}+z_{-\alpha}$ where $z_{2 \alpha} \in A_{2 \alpha}$, $z_{-\alpha} \in A_{-\alpha}$, then using Lemma 2.13 and the fact $J\left(u, A_{-\alpha}, A_{2 \alpha}\right)=0$ we have

$$
0=J(u, x y, w)=J\left(u, z_{2 \alpha}, w\right)+J\left(u, z_{-\alpha}, w\right)=J\left(u, z_{2 \alpha}, w\right) ;
$$

that is,

$$
J\left(u, z_{2 \alpha}, A_{2 \alpha}\right)=0 \text {. }
$$

Now since $z_{2 \alpha} \in A_{2 \alpha}$ we also have by (2.4) $J\left(u, z_{2 \alpha}, A_{\beta}\right)=0$ if $\beta \neq 2 \alpha$. Combining these results, $J\left(u, z_{2 \alpha}, A\right)=\sum_{\beta} J\left(u, z_{2 \alpha}, A_{\beta}\right)=0$ and therefore $z_{2 \alpha} u \in N=0$ by (1.11) and (1.12). Thus $0=z_{2 \alpha} R_{u}$ and therefore $z_{2 \alpha} \in A_{0} \cap A_{2 \alpha}=0$ and this proves

$$
A_{\alpha}^{2} \subset A_{-\alpha} .
$$

Also note that we now have

$$
J\left(A_{0}, A_{\alpha}, A_{\alpha}\right) \subset A_{-\alpha} .
$$

3. More identities. Let $A=A_{0} \oplus A_{\alpha} \oplus \cdots \oplus A_{\gamma}$ be the decomposition of $A$ into a weight space direct sum relative to $R_{u}$ and suppose that for weights $\alpha, \beta, \gamma$ of $R_{u}, \beta \neq \gamma$ and $\beta+\gamma \neq \alpha$. Then for $x \in A_{\alpha}$, $y \in A_{\beta}$ and $z \in A_{\gamma}$ we have by (1.9) and (2.4)

$$
J(x u, y, z)=x J(u, y, z)+J(x, y, z) u-2 J(y z, x, u)=J(x, y, z) u
$$

and therefore $J\left(x\left(R_{u}-\alpha I\right), y, z\right)=J(x, y, z)\left(R_{u}-\alpha I\right)$. By induction we have $J\left(x\left(R_{u}-\alpha I\right)^{n}, y, z\right)=J(x, y, z)\left(R_{u}-\alpha I\right)^{n}$ and hence

$$
J\left(A_{\alpha}, A_{\beta}, A_{\gamma}\right) \subset A_{\alpha} \text { if } \beta \neq \gamma \text { and } \beta+\gamma \neq \alpha .
$$

By the symmetry of the $\alpha, \beta$ and $\gamma$ we may also conclude

$$
\begin{array}{ll}
J\left(A_{\beta}, A_{\gamma}, A_{\alpha}\right) \subset A_{\beta} & \text { if } \gamma \neq \alpha \text { and } \gamma+\alpha \neq \beta \\
J\left(A_{\gamma}, A_{\alpha}, A_{\beta}\right) \subset A_{\gamma} & \text { if } \alpha \neq \beta \text { and } \alpha+\beta \neq \gamma .
\end{array}
$$

Now assume $\alpha \neq \beta \neq \gamma \neq \alpha$. Suppose $\beta+\gamma=\alpha$. If $\gamma+\alpha=\beta$, 
then $\gamma=0$ and therefore $\alpha=\beta$, a contradiction. Therefore $\gamma+\alpha \neq \beta$ and by (3.2) $J\left(A_{\beta}, A_{\gamma}, A_{\alpha}\right) \subset A_{\beta}$. Similarly if $\alpha+\beta=\gamma$, then $\beta=0$ and $\alpha=\gamma$, a contradiction. Therefore $\alpha+\beta \neq \gamma$ and by (3.3) $J\left(A_{\gamma}, A_{\alpha}, A_{\beta}\right) \subset A_{\gamma}$. Thus we have $J\left(A_{\alpha}, A_{\beta}, A_{\gamma}\right) \subset A_{\gamma} \cap A_{\beta}=0$ if $\alpha \neq$ $\beta \neq \gamma \neq \alpha$ and $\beta+\gamma=\alpha$.

With the assumption $\alpha \neq \beta \neq \gamma \neq \alpha$, suppose now that $\beta+\gamma \neq \alpha$. Then by (3.1), $J\left(A_{\alpha}, A_{\beta}, A_{\gamma}\right) \subset A_{\alpha}$. We next note that it is impossible to have $\gamma+\alpha=\beta$ and $\alpha+\beta=\gamma$. So using (3.2) or (3.3) together with $J\left(A_{\alpha}, A_{\beta}, A_{\gamma}\right) \subset A_{\alpha}$ we conclude $J\left(A_{\alpha}, A_{\beta}, A_{\gamma}\right)=0$. Thus we can conclude, using the preceding paragraph,

$$
J\left(A_{\alpha}, A_{\beta}, A_{\gamma}\right)=0 \quad \text { if } \alpha \neq \beta \neq \gamma \neq \alpha .
$$

Now assume two weights are equal, that is, $\alpha=\beta$. Suppose $\gamma \neq$ $0, \alpha,-\alpha$ or $2 \alpha$, then

$$
\begin{aligned}
J\left(A_{\alpha}, A_{\alpha}, A_{\gamma}\right) & \subset A_{\alpha}^{2} A_{\gamma}+A_{\alpha} A_{\gamma} \cdot A_{\alpha}+A_{\gamma} A_{\alpha} \cdot A_{\alpha} \\
& \subset A_{-\alpha} A_{\gamma}+A_{\alpha+\gamma} A_{\alpha} \\
& \subset A_{-\alpha+\gamma} \oplus A_{\gamma+2 \alpha} .
\end{aligned}
$$

However using (3.1) $J\left(A_{\alpha}, A_{\alpha}, A_{\gamma}\right) \subset A_{\alpha}$ and therefore $J\left(A_{\alpha}, A_{\alpha}, A_{\gamma}\right) \subset$ $A_{\alpha} \cap\left(A_{-\alpha+\gamma} \oplus A_{\gamma+2 \alpha}\right)=0$. This proves

$$
J\left(A_{\alpha}, A_{\alpha}, A_{\gamma}\right)=0 \text { if } \gamma \neq 0, \alpha \text {, or }-\alpha 2 \alpha .
$$

For the "exceptional" cases we have

$$
\begin{aligned}
& J\left(A_{\alpha}, A_{\alpha}, A_{\alpha}\right) \subset A_{\alpha}^{2} \cdot A_{\alpha} \subset A_{-\alpha} A_{\alpha} \subset A_{0} . \\
& J\left(A_{\alpha}, A_{\alpha}, A_{0}\right) \subset A_{\alpha}^{2} A_{0}+A_{\alpha} A_{0} \cdot A_{\alpha} \subset A_{-\alpha} . \\
& J\left(A_{\alpha}, A_{\alpha}, A_{-\alpha}\right) \subset A_{\alpha}^{2} A_{-\alpha}+A_{\alpha} A_{-\alpha} \cdot A_{\alpha} \subset A_{\alpha} . \\
& J\left(A_{\alpha}, A_{\alpha}, A_{2 \alpha}\right)=0 .
\end{aligned}
$$

To prove (3.9) let $x, y \in A_{\alpha}, z \in A_{2 \alpha}$, then by (1.9), (2.5) and (2.4)

$$
\begin{aligned}
J(x u, y, z) & =x J(u, y, z)+J(x, y, z) u-2 J(y z, x, u) \\
& =J(x, y, z) u
\end{aligned}
$$

and as usual we have $J\left(x\left(R_{u}-\alpha I\right)^{n}, y, z\right)=J(x, y, z)\left(R_{u}-\alpha I\right)^{n}$. Therefore $J(x, y, z) \in A_{\alpha}$. However by (1.7) $J(x, y, u z)+J(u, y, x z)=$ $J(x, y, z) u+J(u, y, z) x$ and using (2.4) we obtain $J(x, y, u z)=J(x, y, z) u$. This yields $J\left(x, y, z\left(2 \alpha I-R_{u}\right)^{n}\right)=J(x, y, z)\left(2 \alpha I+R_{u}\right)^{n}$ and therefore $J(x, y, z) \in A_{-2 \alpha}$. Combining the above results we have $J(x, y, z) \in A_{\alpha} \cap$ $A_{-2 \alpha}=0$ if $\alpha \neq 0$.

We shall now show $A_{\alpha} A_{\beta}=0$ if $\alpha \neq 0$ and $\beta \neq 0, \pm \alpha$. Let $\alpha$ and $\beta$ be fixed weights of $R_{u}$ and assume $\beta \neq k \alpha, k=0, \pm 1, \pm 2, \cdots$, with 
$\alpha \neq 0$. Then for any other weight $\gamma$ we have by (3.4) $J\left(A_{\beta}, A_{\alpha}, A_{\gamma}\right)=$ 0 if $\beta \neq \alpha \neq \gamma \neq \beta$. However $\alpha \neq \beta$ and therefore $J\left(A_{\beta}, A_{\alpha}, A_{\gamma}\right)=$ if $\alpha \neq \gamma \neq \beta$. Suppose $\gamma=\alpha$, then by (3.5) and the choice of $\beta$, $J\left(A_{\beta}, A_{\alpha}, A_{\alpha}\right)=0$. Suppose $\gamma=\beta$, then $J\left(A_{\beta}, A_{\alpha}, A_{\beta}\right)=J\left(A_{\beta}, A_{\beta}, A_{\alpha}\right)=$ 0 if $\alpha \neq 0, \beta,-\beta$ or $2 \beta$. We know $\alpha \neq 0, \beta$ or $-\beta$ so if $\alpha=2 \beta$, then by (3.9) $J\left(A_{\beta}, A_{\beta}, A_{\alpha}\right)=0$. Combining all these cases we have shown $J\left(A_{\beta}, A_{\alpha}, A_{\gamma}\right)=0$ for any weight $\gamma$ and therefore $J\left(A_{\beta}, A_{\alpha}, A\right)=$ $\sum_{\gamma} J\left(A_{\beta}, A_{\alpha}, A_{\gamma}\right)=0$. By (1.11) and (1.12) $A_{\alpha} A_{\beta} \subset N=0$. This proves

$$
A_{\alpha} A_{\beta}=0 \text { if } \alpha \neq 0 \text { and } \beta \neq k \alpha, k=0, \pm 1, \pm 2, \cdots \text {. }
$$

We now assume $\alpha \neq 0$ and $\beta=k \alpha$ for $k \neq 0, \pm 1$, then $J\left(A_{\alpha}, A_{\beta}, A_{\gamma}\right)=$ $J\left(A_{\alpha}, A_{k \alpha}, A_{\gamma}\right)=0$ if $\alpha \neq k \alpha \neq \gamma \neq \alpha$, by (3.4). But since $k \neq 1$ we have $J\left(A_{\alpha}, A_{k \alpha}, A_{\gamma}\right)=0$ if $\alpha \neq \gamma \neq k \alpha$. Suppose $\gamma=\alpha$, then using (3.5)

$$
\begin{aligned}
J\left(A_{\alpha}, A_{\beta}, A_{\gamma}\right) & =J\left(A_{\alpha}, A_{k \alpha}, A_{\gamma}\right) \\
& =J\left(A_{\alpha}, A_{k \alpha}, A_{\alpha}\right) \\
& =J\left(A_{\alpha}, A_{\alpha}, A_{k \alpha}\right) \\
& =0
\end{aligned}
$$

if $k \alpha \neq 0, \alpha,-\alpha$ or $2 \alpha$. But by the choice of $k$ we need only consider $k \alpha=2 \alpha$ and in this case $J\left(A_{\alpha}, A_{\alpha}, A_{k \alpha}\right)=0$ by (3.9). Now suppose $\gamma=k \alpha$, then

$$
\begin{aligned}
J\left(A_{\alpha}, A_{\beta}, A_{\gamma}\right) & =J\left(A_{\alpha}, A_{k \alpha}, A_{\gamma}\right) \\
& =J\left(A_{\alpha}, A_{k \alpha}, A_{k \alpha}\right) \\
& =J\left(A_{k \alpha}, A_{k \alpha}, A_{\alpha}\right) \\
& =0
\end{aligned}
$$

if $\alpha \neq 0, k \alpha,-k \alpha$ or $2 k \alpha$, by (3.5). Again by the choice of $k$ and $\alpha$ we need only consider $\alpha=2 k \alpha$. In this case $k=1 / 2$ and therefore $\gamma=\beta=k \alpha=1 / 2 \alpha$. This yields $J\left(A_{\alpha}, A_{\beta}, A_{\gamma}\right)=J\left(A_{\beta}, A_{\beta}, A_{2,3}\right)=0$ by (3.9). Combining all of these cases we have for any weight $\gamma$, $J\left(A_{\alpha}, A_{k \alpha}, A_{\gamma}\right)=0$ if $\alpha \neq 0, k \neq 0, \pm 1$ and as before this gives

$$
A_{\alpha} A_{k \alpha}=0 \text { if } \alpha \neq 0, k \neq 0, \pm 1 \text {. }
$$

(3.10) and (3.11) yield

$$
A_{\alpha} A_{\beta}=0 \text { if } \alpha \neq 0, \beta \neq 0, \pm \alpha .
$$

Since $R_{u}$ is not nilpotent, there exists a weight $\alpha \neq 0$. We shall now show that $-\alpha$ is also a weight of $R_{u}$. For suppose $-\alpha$ is not a weight, then by the usual convention $A_{-\alpha}=0$ and noting that none of the previously derived identities use the fact that $A_{-\alpha} \neq 0$ we have for $\beta \neq 0$ or $\alpha$, that $A_{\alpha} A_{\beta}=0$ by (3.12). For $\beta=0, A_{\alpha} A_{\beta} \subset A_{\alpha}$ and for 
$\beta=\alpha, A_{\alpha} A_{\beta} \subset A_{-\alpha}=0$ using (2.14). Therefore $A_{\alpha}$ is a nonzero ideal of $A$ and so $A=A_{\alpha}$. But $u \in A$ and $u \notin A_{\alpha}=A$, a contradiction. Therefore $-\alpha$ is a weight if $\alpha$ is a weight.

Now set $\mathscr{A}_{\alpha}=A_{\alpha} A_{-\alpha} \oplus A_{\alpha} \oplus A_{-\alpha}$ where $\alpha$ is a nonzero weight. Then $\mathscr{A}_{\alpha} \neq 0$ and for $\beta=0, \pm \alpha$ we have $\mathscr{A}_{\alpha} A_{\beta} \subset \mathscr{A}_{\alpha}$. For $\beta \neq 0, \pm \alpha$ we have $A_{\alpha} A_{\beta}=A_{-\alpha} A_{\beta}=0$ by (3.12). Now by (3.4) and (3.12) we have for $x \in A_{\alpha}, y \in A_{-\alpha}, z \in A_{\beta}$ that $0=J(x, y, z)=x y \cdot z+y z \cdot x+z x \cdot y=$ $x y \cdot z$ and so $0=A_{\alpha} A_{-\alpha} \cdot A_{\beta}$. Thus in all cases $\mathscr{A}_{\alpha} A_{\beta} \subset \mathscr{A}_{\alpha}$ and therefore $\mathscr{A}_{\alpha}$ is a nonzero ideal of $A$ and we have $A=\mathscr{A}_{\alpha}$. This proves

Proposition 3.13. If $A$ is a finite dimensional simple non-Lie Malcev algebra over an algebraically closed field of characteristic not 2 or 3 and $A$ contains an element $u$ such that $R_{u}$ is not a nilpotent linear transformation, then there exists an $\alpha \neq 0$ such that $A=A_{0} \oplus A_{\alpha} \oplus A_{-\infty}$ where $A_{a}=\left\{x \in A: x\left(a I-R_{u}\right)^{k}=0\right.$ for some $\left.k>0\right\}$ and $A_{0}=A_{\alpha} A_{-\alpha}$.

4. A decomposition of $A$ relative to $A_{0}$. Let us consider the decomposition of $A$ as given Proposition 3.13; that is,

$$
A=A_{0} \oplus A_{a} \oplus A_{-a} .
$$

For any $y_{0}, z_{0} \in A_{0}$ and $x \in A_{a}(a=0, \pm \alpha)$, we use (2.8) and (2.11) to see that

$$
0=J\left(x, y_{0}, z_{0}\right)=x\left(R_{y_{0}} R_{z_{0}}-R_{z_{0}} R_{y_{0}}\right)
$$

Therefore,

$$
R\left(A_{0}\right) \equiv\left\{R_{x_{0}}: x_{0} \in A_{0}\right\}
$$

is a commuting set of linear transformations acting on $A_{a}$. We can find $R\left(A_{0}\right)$-invariant subspaces $M_{\lambda}(\alpha)$ [2; Chapter 4] such that

$$
A_{a}=\sum_{\lambda} \oplus M_{\lambda}(a) \quad(a=0, \pm \alpha),
$$

where on each $M_{\lambda}(a)$ the transformation $R_{x_{0}}$, for any $x_{0} \in A_{0}$, has a matrix of the form

$$
\left[\begin{array}{cc}
\lambda\left(x_{0}\right) & 0 \\
* & \lambda\left(x_{0}\right)
\end{array}\right]
$$

that is, $M_{\lambda}(\alpha)$ has a basis $\left\{x_{1}, x_{2}, \cdots, x_{m}\right\}(m=m(\lambda, \alpha))$ such that for any $x_{0} \in A_{0}$, there exists $a_{i j}\left(x_{0}\right) \in F$ for which

$$
x_{i} R_{x_{0}}=\sum_{j=1}^{i-1} a_{i j}\left(x_{0}\right) x_{j}+\lambda\left(x_{0}\right) x_{i},
$$

where $\lambda\left(x_{0}\right) \in F$ and, of course, $i=1,2, \cdots, m$. 
Using the usual terminology we call the function $\lambda$ defined by $\lambda: x_{0} \rightarrow \lambda\left(x_{0}\right)$ a weight of $A_{0}$ in $A_{a}$ or just a weight and the corresponding $M_{\lambda}(\alpha)$ a weight space of $A_{a}$ corresponding to $\lambda$ or just a weight space of $A_{a}$. It is easily seen [2] that $A_{a}$ has finitely many weights and the weights are linear functionals on $A_{0}$ to $F$. Also

$$
\begin{array}{r}
M_{\lambda}(a)=\left\{x \in A_{a}: \text { for all } x_{0} \in A_{0}, x\left(R_{x_{0}}-\lambda\left(x_{0}\right) I\right)^{k}=0\right. \\
\text { for some integer } k>0\}
\end{array}
$$

and for this weight $\lambda$ we have $\lambda(u)=a$. For suppose $\lambda(u)=b$, then there exists an $x \neq 0$ in $M_{\lambda}(a)$ such that $b x=x R_{u}$. But $M_{\lambda}(a) \subset A_{a}=$ $\left\{x \in A: x\left(R_{u}-a I\right)^{n}=0\right\}$; therefore $(b-a) x=x\left(R_{u}-a I\right)$ and by induction $(b-a)^{n} x=x\left(R_{u}-a I\right)^{n} \quad$ so for some integer $N, \quad(b-a)^{N} x=$ $x\left(R_{u}-a I\right)^{N}=0$ and thus $a=b=\lambda(u)$. We now combine the weight space decompositions of the $A_{a}$ to form a weight space decomposition of $A$ in

Proposition 4.2. Let $A=A_{0} \oplus A_{\alpha} \oplus A_{-\alpha}$ be a simple Malcev algebra as determined by Proposition 3.13, then we can write $A=A_{0} \oplus$ $\sum_{\lambda} \oplus M_{\lambda}(\alpha) \oplus \sum_{\mu} \oplus M_{\mu}(-\alpha)$ where all weights are distinct and any nonzero weight $\rho$ of $A_{0}$ in $A$ is a weight of $A_{0}$ in $A_{\alpha}$ or $A_{-\alpha}$ but not both.

Proof. The first part is clear noting that in the original weight space decomposition $A_{a}=\sum_{\gamma} \oplus M_{\gamma}(a)$ the weights of $A_{0}$ in $A_{a}$ can be taken to be distinct. Also if $\lambda$ is a weight of $A_{0}$ in $A_{\alpha}$ and $\mu$ a weight of $A_{0}$ in $A_{-\alpha}$, then $\lambda(u)=\alpha \neq-\alpha=\mu(u)$ and therefore $\lambda \neq \mu$. Now let $\rho \neq 0$ be any weight of $A_{0}$ in $A$ with weight space $M_{\rho}=$ $\left\{x \in A: x\left(R_{x_{0}}-\rho\left(x_{0}\right) I\right)^{k}=0\right\}$ and let $y=y_{0}+y_{\alpha}+y_{-\alpha} \in M_{\rho}$ where $y_{a} \in A_{a}$ with $a=0, \pm \alpha$. Then for some integer $N>0$,

$$
\begin{aligned}
0= & y\left(R_{x_{0}}-\rho\left(x_{0}\right) I\right)^{N} \\
= & y_{0}\left(R_{x_{0}}-\rho\left(x_{0}\right) I\right)^{N} \\
& +y_{\alpha}\left(R_{x_{0}}-\rho\left(x_{0}\right) I\right)^{N}+y_{-\alpha}\left(R_{x_{0}}-\rho\left(x_{0}\right) I\right)^{N}
\end{aligned}
$$

and by the uniqueness of the decomposition $A=A_{0} \oplus A_{\alpha} \oplus A_{-\alpha}$ we have $y_{a}\left(R_{x_{0}}-\rho\left(x_{0}\right) I\right)^{N}=0$ for $a=0, \pm \alpha$. Now by using the binomial theorem and $A_{0}^{2}=0$ we have $0=y_{0}\left(R_{x_{0}}-\rho\left(x_{0}\right) I\right)^{N}=y_{0} \rho\left(x_{0}\right)^{N}$ and since $\rho \neq 0, y_{0}=0$. Thus we have $y_{a}\left(R_{x_{0}}-\rho\left(x_{0}\right) I\right)^{N}=0, a= \pm \alpha$, for some integer $N$ and so $\rho$ is a weight of $A_{0}$ in $A_{\alpha}$ and $A_{-\alpha}$. Now suppose $y_{\alpha}$ and $y_{-\alpha}$ are both nonzero, then since $\rho$ is a weight of $A_{0}$ in $A_{\alpha}, \rho(u)=$ $\alpha$ and since $\rho$ is a weight of $A_{0}$ in $A_{-\alpha}, \rho(u)=-\alpha$, a contradiction. Thus $\rho$ is a weight of $A_{0}$ in either $A_{\alpha}$ or $A_{-\alpha}$ but not both.

We shall use the usual convention that if $\rho$ is not a weight of $A_{0}$ in $A$, then $M_{\rho}=0$. Let $M_{\lambda}(\alpha)$ and $M_{\mu}(\alpha)$ be weight spaces of $A_{0}$ in $A_{a}$ 
and let $x_{0}, y_{0} \in A_{0}$ and $x \in M_{\lambda}(a), y \in M_{\mu}(a)$, then using (2.8) and (1.7) we have

$$
\begin{aligned}
J\left(x, x_{0}, y_{0} y\right) & =J\left(y_{0}, x_{0}, x y\right)+J\left(x, x_{0}, y_{0} y\right) \\
& =J\left(y_{0}, x_{0}, y\right) x+J\left(x, x_{0}, y\right) y_{0} \\
& =J\left(x, x_{0}, y\right) y_{0} .
\end{aligned}
$$

Thus $J\left(x_{0}, x, y\left(R_{y_{0}}-\mu\left(y_{0}\right) I\right)\right)=-J\left(x_{0}, x, y\right)\left(R_{y_{0}}+\mu\left(y_{0}\right) I\right)$ and by induction

$$
J\left(x_{0}, x, y\left(R_{y_{0}}-\mu\left(y_{0}\right) I\right)^{n}\right)=(-1)^{n} J\left(x_{0}, x, y\right)\left(R_{y_{0}}+\mu\left(y_{0}\right) I\right)^{n} .
$$

From this we obtain $J\left(x_{0}, x, y\right) \in M_{-\mu}(-a)$ and interchanging the roles of $x$ and $y$ we see $J\left(x_{0}, x, y\right) \in M_{-\lambda}(-a)$; this proves

$$
J\left(A_{0}, M_{\lambda}(a), M_{\mu}(a)\right) \subset M_{-\lambda}(-a) \cap M_{-\mu}(-a) .
$$

From (4.3) we obtain

$$
\begin{gathered}
J\left(A_{0}, M_{\lambda}(a), M_{\lambda}(a)\right) \subset M_{-\lambda}(-a) \\
J\left(A_{0}, M_{\lambda}(a), M_{\mu}(a)\right)=0 \text { if } \lambda \neq \mu .
\end{gathered}
$$

We shall next show

$$
M_{\lambda}(a) M_{\mu}(a)=0 \text { if } \lambda \neq \mu .
$$

For let $x_{0} \in A_{0}, x \in M_{\lambda}(a)$ and $y \in M_{\mu}(a)$, then by (4.5) $0=J\left(x, y, x_{0}\right)$ and therefore $x y R_{x_{0}}=x R_{x_{0}} \cdot y+x \cdot y R_{x_{0}}$ and hence $x y\left(R_{x_{0}}-\left(\mu\left(x_{0}\right)+\lambda\left(x_{0}\right)\right) I\right)=$ $x\left(R_{x_{0}}-\lambda\left(x_{0}\right) I\right) \cdot y+x \cdot y\left(R_{x_{0}}-\mu\left(x_{0}\right) I\right)$. In the usual way we can prove there exists an integer $N$ such that $x y\left(R_{x_{0}}-\left(\mu\left(x_{0}\right)+\lambda\left(x_{0}\right)\right) I\right)^{N}=0$ and since we know $x y \in A_{-a}$ this shows $x y \in M_{\lambda+\mu}(-a)$ if $\lambda+\mu$ (defined by $\left.(\lambda+\mu)\left(x_{0}\right)=\lambda\left(x_{0}\right)+\mu\left(x_{0}\right)\right)$ is a weight of $A_{0}$ in $A_{-a}$, or $x y=0$. If $x y \neq 0$, then $\lambda+\mu$ is a weight of $A_{0}$ in $A_{-a}$ where $\lambda$ and $\mu$ are weights of $A_{0}$ in $A_{a}$ and therefore $-a=(\lambda+\mu)(u)=\lambda(u)+\mu(u)=a+a$, a contradiction.

Next we have for any weight $\lambda$ of $A_{0}$ in $A_{a}$

$$
M_{\lambda}(a) M_{\lambda}(a) \subset M_{-\lambda}(-a)
$$

if $-\lambda$ is a weight of $A_{0}$ in $A_{-a}$. For let $x_{0} \in A_{0}$ and $\lambda \equiv \lambda\left(x_{0}\right) \in F$ and let $M_{\lambda}(a)$ have basis $\left\{x_{1}, \cdots, x_{m}\right\}$ as in (4.1). Then using (1.2) we obtain

$$
\begin{aligned}
\lambda^{2} x_{1} x_{2} & =\lambda x_{1}\left(\lambda x_{2}+a_{21} x_{1}\right) \\
& =x_{1} R_{x_{0}} \cdot x_{2} R_{x_{0}} \\
& =\left(x_{0} x_{1} \cdot x_{2}\right) x_{0}+\left(x_{1} x_{2} \cdot x_{0}\right) x_{0}+\left(x_{2} x_{0} \cdot x_{0}\right) x_{1} \\
& =-\lambda x_{1} x_{2} R_{x_{0}}+x_{1} x_{2} R_{x_{0}}^{2}+\lambda^{2} x_{2} x_{1}
\end{aligned}
$$

and thus

$$
0=x_{1} x_{2}\left(R_{x_{0}}^{2}-\lambda R_{x_{0}}-2 \lambda^{2} I\right)=x_{1} x_{2}\left(R_{x_{0}}+\lambda I\right)\left(R_{x_{0}}-2 \lambda I\right) .
$$


Now since $\lambda$ is a weight of $A_{0}$ in $A_{a},-2 \lambda$ is not a weight of $A_{0}$ in $A_{-a}:-a=(2 \lambda)(u)=2 \lambda(u)=2 a$. Thus the above equation implies $x_{1} x_{2}\left(R_{x_{0}}+\lambda I\right)=0$ and therefore $x_{1} x_{2} \in M_{-\lambda}(-a)$. Next $x_{1} x_{0} \cdot x_{3} x_{0}=$ $\lambda x_{1}\left(\lambda x_{3}+a_{32} x_{2}+a_{31} x_{1}\right)=\lambda^{2} x_{1} x_{3}+s$ where $s \in M_{-\lambda}(-a)$ and $\left(x_{0} x_{1} \cdot x_{3}\right) x_{0}+$ $\left(x_{1} x_{3} \cdot x_{0}\right) x_{0}+\left(x_{3} x_{0} \cdot x_{0}\right) x_{1}=-\lambda x_{1} x_{3} R_{x_{0}}+x_{1} x_{3} R_{x_{0}}^{2}+\lambda^{2} x_{3} x_{1}+t$ where $t \in M_{-\lambda}(-a)$. Therefore using (1.2) we obtain $0=x_{1} x_{3}\left(R_{x_{0}}+\lambda I\right)\left(R_{x_{0}}-2 \lambda I\right)+w$ where $w \in M_{-\lambda}(-a)$ and actually $w=3 \lambda a_{31} x_{2} x_{1}$. Therefore $0=x_{1} x_{3}\left(R_{x_{n}}+\lambda I\right)^{2}$ $\left(R_{x_{0}}-2 \lambda I\right)$ and as before $x_{1} x_{3}\left(R_{x_{0}}+\lambda I\right)^{2}=0$ so that $x_{1} x_{3} \in M_{-\lambda}(-a)$. Continuing this process we obtain $x_{1} x_{k} \in M_{-\lambda}(-a)$ for $k=1,2, \cdots, m$. Next consider the product $x_{2} x_{3}$.

$$
\begin{aligned}
x_{2} x_{0} \cdot x_{3} x_{0} & =\left(\lambda x_{2}+a_{21} x_{1}\right)\left(\lambda x_{3}+a_{32} x_{2}+a_{31} x_{1}\right) \\
& =\lambda^{2} x_{2} x_{3}+s
\end{aligned}
$$

where $s \in M_{-\lambda}(-a)$ and

$$
\left(x_{0} x_{2} \cdot x_{3}\right) x_{0}+\left(x_{2} x_{3} \cdot x_{0}\right) x_{0}+\left(x_{3} x_{0} \cdot x_{0}\right) x_{2}=x_{2} x_{3}\left(R_{x_{0}}^{2}-\lambda R_{x_{0}}-\lambda^{2} I\right)+t
$$

where $t \in M_{-\lambda}(-a)$, therefore $0=x_{2} x_{3}\left(R_{x_{0}}+\lambda I\right)\left(R_{x_{0}}-2 \lambda I\right)+w$ where $w \in M_{-\lambda}(-\alpha)$. Therefore for some integer $k>0$ such that $w\left(R_{x_{0}}+\lambda I\right)^{k}=$ 0 we have $0=x_{2} x_{3}\left(R_{x_{0}}+\lambda I\right)^{k+1}\left(R_{x_{0}}-2 \lambda I\right)$ and as before $x_{2} x_{3} \in M_{-\lambda}(-a)$. We continue this process showing $x_{2} x_{k} \in M_{-\lambda}(-a)$ and in general $x_{i} x_{j} \in M_{-\lambda}(-a)$ for $i, j=1, \cdots, m$. This completes the proof of (4.7).

We now show

$$
M_{\lambda}(a) \cdot M_{\mu}(-a)=0 \text { if } \lambda+\mu \neq 0 .
$$

By (2.7) we have for $x \in M_{\lambda}(a), y \in M_{\mu}(-a)$ and $x_{0} \in A_{0}$ that $0=$ $J\left(x, y, x_{0}\right)$ and as usual we obtain $x y\left(R_{x_{0}}-\left(\lambda\left(x_{0}\right)+\mu\left(x_{0}\right)\right) I\right)^{N}=0$ for some integer $N>0$. Now $z=x y \in A_{0}$ and suppose $z \neq 0$, then, since $\lambda+\mu \neq 0, \lambda+\mu$ is a nonzero weight of $A_{0}$ in $A_{0}$, a contradiction to Proposition 4.2.

Let $x \in M_{\rho}(a), y \in M_{\lambda}(a)$ and $z \in M_{\mu}(-a)$, then using (1.9), (2.7) and (2.8) we have

$$
\begin{aligned}
J\left(x x_{0}, y, z\right) & =x J\left(x_{0}, y, z\right)+J(x, y, z) x_{0}-2 J\left(y z, x, x_{0}\right) \\
& =J(x, y, z) x_{0}
\end{aligned}
$$

and therefore $J\left(x\left(R_{x_{0}}-\rho\left(x_{0}\right) I\right), y, z\right)=J(x, y, z)\left(R_{x_{0}}-\rho\left(x_{0}\right) I\right)$ and as usual we obtain $J(x, y, z) \in M_{\mathrm{\rho}}(a)$. Interchanging $x$ and $y$ we also obtain $J(x, y, z) \in M_{\lambda}(a)$ and therefore $J(x, y, z) \in M_{\lambda}(a) \cap M_{\rho}(a)=0$ if $\lambda \neq \rho$. Now assume $\lambda \neq \rho$ and assume $\mu=-\lambda$ is a weight of $A_{0}$ in $A_{-a}$, then

$$
0=J(x, y, z)=x y \cdot z+y z \cdot x+z x \cdot y=y z \cdot x,
$$

using (4.6) and (4.8). This proves 


$$
M_{\lambda}(a) M_{-\lambda}(-a) \cdot M_{\rho}(a)=0
$$

if $\lambda \neq \rho$ are weights of $A_{0}$ in $A_{a}$ such that $-\lambda$ is a weight of $A_{0}$ in $A_{-a}$.

We shall now show if $\lambda$ is a nonzero weight of $A_{0}$ in $A_{a}$ with weight space $M_{\lambda}(a)$, then $-\lambda$ is a nonzero weight of $A_{0}$ in $A_{-a}$ with weight space $M_{-\lambda}(-a)$. The proof is similar to that following (3.12): Suppose $-\lambda$ is not a weight of $A_{0}$ in $A_{-a}$, then $M_{-\lambda}(-a)=0 ; M_{\lambda}(a) M_{\lambda}(a)=$ $0 ; \quad M_{\lambda}(a) M_{\rho}(a)=0$ if $\rho \neq \lambda ; A_{0} M_{\lambda}(a) \subset M_{\lambda}(a)$ and $M_{\lambda}(\alpha) M_{\mu}(-\alpha)=0$ if $\mu+\lambda \neq 0$. Thus $M_{\lambda}(a)$ is a proper ideal of $A$, a contradiction.

Set $M_{\lambda}=M_{\lambda}(\alpha) M_{-\lambda}(-\alpha) \oplus M_{\lambda}(\alpha) \oplus M_{-\lambda}(-\alpha)$ for some nonzero weight $\lambda$ of $A_{0}$ in $A_{\alpha}$. Then analogous to Proposition 3.13, $M_{\lambda}$ can be shown to be a nonzero ideal of $A$ and we have

Proposition 4.10. If $A=A_{0} \oplus A_{\alpha} \oplus A_{-\alpha}$ is a simple Malcev algebra as determined by Proposition 3.13, then there exists a nonzero weight $\lambda$ of $A_{0}$ in $A$ with weight space $M_{\lambda}(\alpha)=A_{\alpha}$ and such that $-\lambda$ is a weight of $A_{0}$ in $A$ with weight space $M_{-\lambda}(-a)=A_{-\alpha}$.

We shall identify $\alpha$ with $\lambda$ as a weight, that is, use the notation $\alpha\left(x_{0}\right)$ for $\lambda\left(x_{0}\right)$ and also identify $M_{\lambda}(\alpha)=A_{\alpha}, M_{-\lambda}(-\alpha)=A_{-\alpha}$. Note that Proposition 4.10 implies there exists a basis for $A$ so that for every $x \in A_{0}, R_{x}$ has a matrix of the form

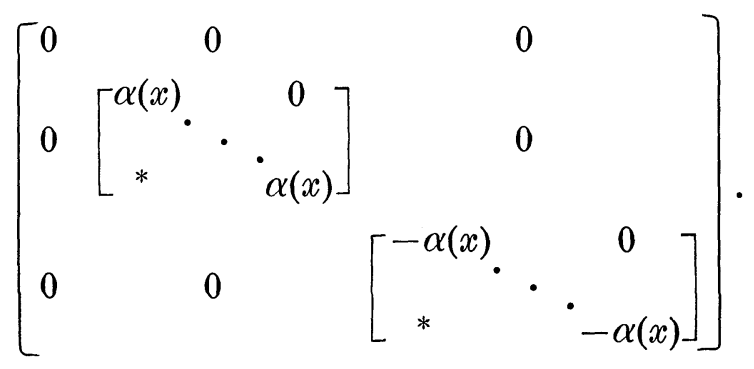

5. The trace form. Set $(x, y)=$ trace $R_{x} R_{y}$, then it is shown [3] that this is actually an invariant form; that is $(x, y)$ is a bilinear form on $A$ such that for all $x, y, z \in A,(x y, z)=(x, y z)$. Also a bilinear form $(x, y)$ is nondegenerate on $A$ if $(x, y)=0$ for all $y \in A$ implies $x=0$.

Theorem 5.1. If $A=A_{0} \oplus A_{\alpha} \oplus A_{-\alpha}$ is a finite dimensional simple non-Lie Malcev algebra over an algebraically closed field of characteristic zero and if $A$ contains an element $u$ such that $R_{u}$ is not nilpotent, then $(x, y)=$ trace $R_{x} R_{y}$ is a nondegenerate invariant form on $A$ and dimension $A_{\alpha}=$ dimension $A_{-\alpha}$.

Proof. On $A=A_{0} \oplus A_{\alpha} \oplus A_{-\alpha} R_{u}$ has the matrix 


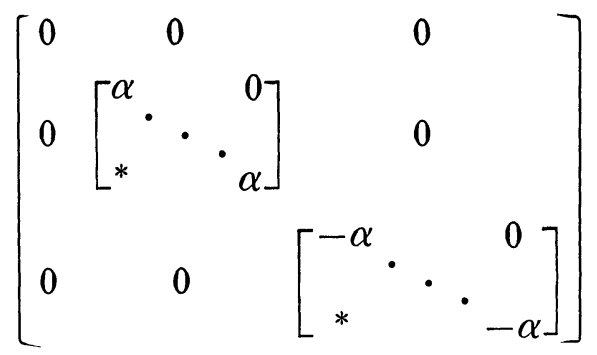

and since $u \in A=J(A, A, A)$ (by 1.12) we have by [3; 2.12] that $0=$ trace $R_{u}=\alpha\left(n_{\alpha}-n_{-\alpha}\right)$ where $n_{a}=$ dimension $A_{a}, a= \pm \alpha$.

Now to show $(x, y)$ is nondegenerate, let $T=\{x \in A:(x, A)=0\}$ where for subsets $B, C$ of $A$ we set $(B, C)=\{(b, c): b \in B, c \in C\}$ and for $x \in A,(x, C)=\{(x, c): c \in C\}$. Since $(x, y)$ is an invariant form on $A, T$ is an ideal of $A$ and since $A$ is simple, $T=0$ or $T=A$. If $T=$ $A$, then $(A, A)=0$ and from the matrix of $R_{u}$ we see that

$$
0=(u, u)=\operatorname{trace} R_{u}^{2}=2 n \alpha^{2}
$$

where $n=$ dimension $A_{\alpha}$. Since $F$ is of characteristic zero, $\alpha=0$, a contradiction. Thus $T=0$ which implies $(x, y)$ is nondegenerate on $A$.

Corollary 5.2. If $A=A_{0} \oplus A_{\alpha} \oplus A_{-\alpha}$ is a simple Malcev algebra as above then

$$
\left(A_{0}, A_{\alpha}\right)=\left(A_{0}, A_{-\alpha}\right)=\left(A_{\alpha}, A_{\alpha}\right)=\left(A_{-\alpha}, A_{-\alpha}\right)=0 .
$$

Proof. Since $R_{u}$ is nonsingular on $A_{a}, a \neq 0, A_{a}=A_{a} R_{u}$. Therefore $\left(A_{0}, A_{a}\right)=\left(A_{0}, A_{a} R_{u}\right)=\left(A_{0} R_{u}, A_{a}\right)=0$, the second equality uses $(x, y)$ is an invariant form and the third uses (2.11). Also $\left(A_{a}, A_{a}\right)=\left(u A_{a}, A_{a}\right)=$ $\left(u, A_{a} A_{a}\right) \subset\left(u, A_{-a}\right)=0$.

CoRollary 5.3. If $A_{0}^{*}$ is the dual space of $A_{0}$ consisting of linear functionals on $A_{0}$ and $f \in A_{0}^{*}$, then $f=c \alpha$ for some $c \in F$.

Proof. First, $(x, y)$ is nondegenerate on $A_{0}$. For if $x_{0} \in A_{0}$ is such that $\left(x_{0}, A_{0}\right)=0$, then

$$
\begin{aligned}
\left(x_{0}, A\right)= & \left(x_{0}, A_{0} \oplus A_{\alpha} \oplus A_{-\alpha}\right) \\
& \subset\left(x_{0}, A_{0}\right)+\left(x_{0}, A_{\alpha}\right)+\left(x_{0}, A_{-\alpha}\right) \\
= & 0
\end{aligned}
$$

by the preceding corollary and therefore $x_{0}=0$ by Theorem 5.1. Now if $f \in A_{0}^{*}$, then there exists a unique element [2, page 141] $a_{f} \in A_{0}$ 
such that for all $x \in A_{0}, f(x)=\left(x, a_{f}\right)=\operatorname{trace} R_{x} R_{a_{f}}=$

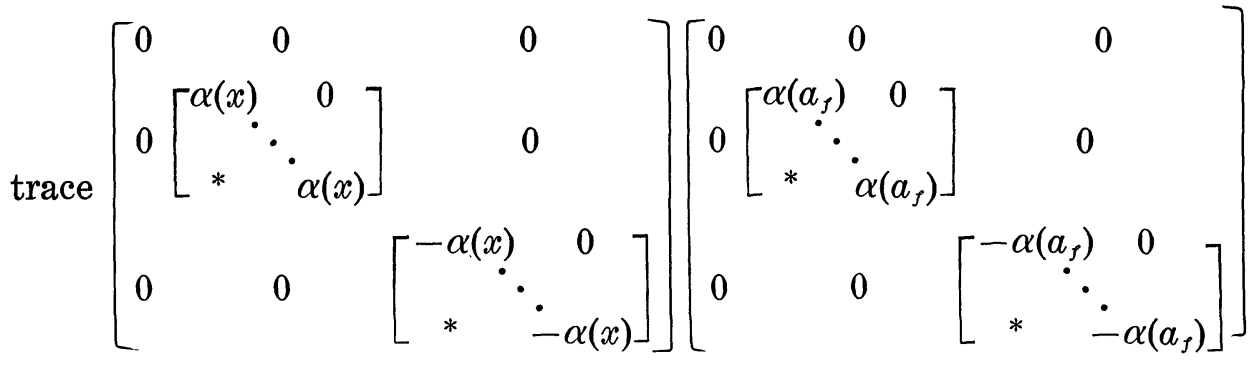

$=2 n \alpha\left(a_{f}\right) \alpha(x)$; using the remarks at the end of $\S 4$ to obtain the form of the matrices of $R_{x}$ and $R_{\alpha_{f}}$. Thus $f=c \alpha$ where $c=2 n \alpha\left(\alpha_{f}\right) \in F$.

Corollary 5.4. The dimension of $A_{0}$ is one.

Proof. $\quad 0<\operatorname{dimension} A_{0}=\operatorname{dimension} A_{0}^{*}=\operatorname{dimension} u F=1$.

We shall frequently refer to a Malcev algebra $A$ that satisfies Theorem 5.1 as a "usual simple non-Lie Malcev algebra" and for the remainder of this paper we shall assume the algebraically closed field $F$ is of characteristic zero.

6. The diagonalization of $R_{u}$. Using Proposition 4.10 and Corollary 5.4 we are able to decompose $A$ relative to $R\left(A_{0}\right)$ into the form

$$
A=A_{0} \oplus A_{\alpha} \oplus A_{-\alpha}
$$

where $A_{0}=u F$. From this the matrix of $R_{u}$ on $A_{a}, a= \pm \alpha$, has the form

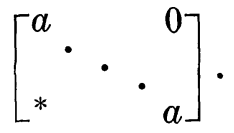

We shall show in this section that $R_{u}$ can be diagonalized. Put $R_{u}$ into its Jordan canonical form on $A_{a}$, that is, find $R_{u}$-invariant subspaces $U_{i}(a)$ of $A_{a}$ such that $A_{a}=U_{1}(a) \oplus \cdots \oplus U_{m_{a}}(a)$ and each $U_{i}(a)$ has a basis $\left\{x_{i 1}, \cdots, x_{i m_{i}}\right\}$ so that the action of $R_{u}$ is given by

$$
\begin{aligned}
x_{i 1} R_{u} & =a x_{i 1} \\
x_{i j} R_{u} & =a x_{i j}+x_{i j-1} \\
j & =2, \cdots, m_{i} .
\end{aligned}
$$

Thus on $U_{i}(a), R_{u}$ has an $m \times m$ matrix of the form

$$
\left[\begin{array}{llllll}
a & & & & 0 \\
1 & a & & & \\
& 1 & & & \\
& \cdot & \cdot & & \\
0 & & & \dot{1} & a
\end{array}\right]
$$


where $m=$ dimension $U_{i}(a)$. We shall now investigate the multiplicative relations between the $U$ 's and show that the dimension of all the $U_{i}(a)$ is one and therefore $R_{u}$ will have a diagonal matrix.

LEMMA 6.2. $U_{i}(a) U_{i}(a)=0$.

Proof. Let $U_{i}(a)$ have basis $\left\{x_{1}, \cdots, x_{m}\right\}$ as given by (6.1). If $m=$ 1 , we are finished. Suppose $m>1$, then using (1.6)

$$
\begin{aligned}
0 & =-J\left(u, x_{2}, x_{2}\right) R_{u} \\
& =J\left(u, x_{2}, x_{2} R_{u}\right) \\
& =a J\left(u, x_{2}, x_{2}\right)+J\left(u, x_{2}, x_{1}\right) \\
& =J\left(u, x_{2}, x_{1}\right) \\
& =x_{2} x_{1} \cdot u+x_{1} u \cdot x_{2}+u x_{2} \cdot x_{1} \\
& =x_{2} x_{1}\left(R_{u}-2 a I\right) .
\end{aligned}
$$

But we know $A_{2 a}=0$, therefore $x_{1} x_{2}=0$. Now using (1.6) we have, in general, for any $i=1, \cdots, m$,

$$
\begin{aligned}
0 & =J\left(u, x_{i}, x_{i} R_{u}\right) \\
& =J\left(u, x_{i}, x_{i-1}\right)+a J\left(u, x_{i}, x_{i}\right) \\
& =J\left(u, x_{i}, x_{i-1}\right)
\end{aligned}
$$

and again using (1.6),

$$
\begin{aligned}
0 & =J\left(u, x_{i}, x_{i-1} R_{u}\right) \\
& =J\left(u, x_{i}, x_{i-2}\right)+a J\left(u, x_{i}, x_{i-1}\right) \\
& =J\left(u, x_{i}, x_{i-2}\right)
\end{aligned}
$$

Continuing this process we have

$$
J\left(u, x_{i}, x_{k}\right)=0
$$

for all $k \leqq i$. Now if $i<k$, then by the preceding sentence

$$
0=J\left(u, x_{k}, x_{i}\right)=J\left(u, x_{i}, x_{k}\right) \text {. }
$$

Thus

$$
J\left(u, x_{i}, x_{k}\right)=0
$$

for all $i, k=1, \cdots, m$.

By linearity this implies

$$
J(u, x, y)=0 \quad \text { for all } x, y \in U_{i}(a) .
$$

Thus

$$
x y R_{u}=x R_{u} \cdot y+\cdot y R_{u}
$$


and

$$
x y\left(R_{u}-2 a I\right)=x\left(R_{u}-a I\right) \cdot y+x \cdot y\left(R_{u}-a I\right)
$$

As usual we can find an $N$ large enough so that $x y\left(R_{u}-2 a I\right)^{N}=0$. But we know $A_{2 a}=0$, therefore $x y=0$.

LEMmA 6.3. Let $x \in A_{a}$ be such that $x R_{u}=a x$ and let $U_{i}(-a) \equiv$ $\left\{y_{1}, \cdots, y_{m}\right\}$, then $x y_{i}=0$ for $i=1, \cdots, m-1$ and $x y_{m}=\lambda u$ where $\lambda=-\left(y_{m}, x\right) / 2 n a$.

Proof. Using the invariant form $(x, y)$ we have $\left(y_{m} x, u\right)=\left(y_{m}, x u\right)=$ $a\left(y_{m}, x\right)$. Since $x y_{m} \in A_{0}=u F$ we may write $x y_{m}=\lambda u$, then $\left(y_{m} x, u\right)=$ $(-\lambda u, u)=-\lambda(u, u)=-\lambda 2 n a^{2}(a= \pm \alpha)$. Thus $\lambda=-\left(y_{m}, x\right) / 2 n a$.

Now since $x \in A_{a}$ and $U_{i}(-a) \subset A_{-a}$, we have by (2.4) and (2.11) that $0=J\left(x, y_{2}, u\right)=x y_{2} \cdot u+y_{2} u \cdot x+u x \cdot y_{2}=\left(-a y_{2}+y_{1}\right) x-a x y_{2}=y_{1} x$. Again $0=J\left(x, y_{3}, u\right)=x y_{3} \cdot u+y_{3} u \cdot x+u x \cdot y_{3}=\left(-a y_{3}+y_{2}\right) x-a x y_{3}=$ $y_{2} x$. Continuing this process we eventually obtain $0=J\left(x, y_{m}, u\right)=$ $x y_{m} \cdot u+y_{m} u \cdot x+u x \cdot y_{m}=y_{m-1} x$.

THeOREM 6.4. Let $x \in A_{a}$ be such that $x R_{u}=a x$ and let $U_{i}(-a)$ be such that $x U_{i}(-a) \neq 0$, then dimension $U_{i}(-a)=1$.

Proof. Let $B=u F \oplus x F \oplus U_{i}(-a)$, then using the preceding lemmas and their notation we see that $B$ is a subalgebra of $A$ and $x y_{m}=\lambda u$ where $\lambda \neq 0$. Now by (2.4) we have $J\left(u, x, y_{m}\right)=0$, therefore by [3; Corollary 4.4] we see that $u, x$ and $y_{m}$ are contained in a Lie subalgebra, $L$, of $A$. However this implies $y_{m} u=-a y_{m}+y_{m-1} \in L$ and therefore $y_{m-1} \in L$; again $y_{m-1} u=-a y_{m-1}+y_{m-2} \in L$ and therefore $y_{m-2} \in L$. Continuing this process we obtain $B=L$ and so $B$ is a Lie subalgebra of $A$. Thus for any $z \in B$,

$$
\begin{aligned}
0 & =J\left(z, x, y_{m}\right) \\
& =z\left(R_{x} R_{y_{m}}-R_{y_{m}} R_{x}-R_{x y_{m}}\right) \\
& =z\left(\left[R_{x}, R_{y_{m}}\right]-\lambda R_{u}\right) .
\end{aligned}
$$

Thus on $B$ we have $\lambda R_{u}=\left[R_{x}, R_{y_{m}}\right]$ and therefore the trace of $R_{u}$ on $B$ is zero. But calculating the trace of $R_{u}$ from its matrix on $B$, we obtain that the trace is $0+a-a m$. Thus $m=1$.

Corollary 6.5. The dimensional of all the $U_{i}(-a), a= \pm \alpha$, is one.

Proof. Suppose there exists $U_{j}(-a) \equiv\left\{y_{1}, \cdots, y_{m}\right\}$ of dimension $m>1$. Then for every $U_{i}(a), y_{1} U_{i}(a)=0$. For if there exists some 
$U_{i}(a)$ such that $y_{1} U_{i}(a) \neq 0$, then by Theorem 6.4 , dimension $U_{i}(a)=1$. But this means there exists $x \in A_{a}$ such that $x R_{u}=a x$ and $0 \neq$ $x y_{1} \in x U_{j}(-a)$; so again by Theorem 6.4 , dimension $U_{j}(-a)=1$, a contradiction. Thus $y_{1} U_{i}(a)=0$ for all $i$ and this implies $y_{1} A_{a}=y_{1}\left(U_{1}(a) \oplus \cdots \oplus\right.$ $\left.U_{m_{a}}(a)\right)=0$. Now from Corollary 5.2 we have, since $y_{1} \in A_{-a},\left(A_{0}, y_{1}\right)=$ $\left(A_{-a}, y_{1}\right)=0$ and using the preceding sentence

$$
\left(A_{a}, y_{1}\right)=\left(A_{a}, y_{1} u\right)=\left(A_{a} y_{1}, u\right)=0 \text {. }
$$

Thus $\left(A, y_{1}\right)=0$ and since $(x, y)$ is nondegenerate on $A, y_{1}=0$, a contradiction.

7. Proof of the theorem. Let $A=A_{0} \oplus A_{\alpha} \oplus A_{-\alpha}$ be the usual simple non-Lie Malcev algebra, then we have just seen that $A_{a}$ is the null space of $R_{u}-a I, a=0, \pm \alpha$. The choice of $\alpha \neq 0$ is fixed but arbitrary. In particular we want to consider the case $\alpha=-2$, then all we must do is consider $u^{\prime}=(-2 / \alpha) u$ and decompose $A$ relative to $R_{u}$ (which is also not nilpotent) to obtain $A=A_{0} \oplus A_{-2} \oplus A_{2}$. However we shall work with a fixed $\alpha$ and normalize when necessary.

Let $a, b \in F$ be any characteristic roots (weights) of $R_{u}$, that is, $a, b=0, \pm \alpha$ with characteristic vectors $x, y \in A$; that is, $a x=x R_{u}$, by $=y R_{u}$ or $x \in A_{a}, y \in A_{b}$, then we have

$$
J(x, \mathrm{y}, u)=x y \cdot u-(a+b) x y \quad \text { where } x \in A_{a}, y \in A_{b} .
$$

Using (2.4) and (7.1) we also have

$$
x y \cdot u=(a+b) x y \text { where } y \in A_{a}, y \in A_{b} \text { and } a \neq b \text {. }
$$

Since $x y \in A_{-a}$ if $x, y \in A_{a}$, we have

$$
x y \cdot u=-a x y \quad \text { where } x, y \in A_{a} .
$$

Combining (7.3) and (7.1) yields

$$
J(x, y, u)=-3 a x y \quad \text { where } x, y \in A_{a} .
$$

Let $x, y, z \in A_{a}$, then using (2.14), (2.4), (1.9) and (7.4) we have

$$
\begin{aligned}
0 & =J(x y, z, u) \\
& =x J(y, z, u)+J(x, z, u) y-2 J(z u, x, y) \\
& =x(-3 a y z)+(-3 a x z) y-2 a J(z, x, y) .
\end{aligned}
$$

Therefore

$$
\begin{aligned}
2 J(x, y, z) & =-3(x \cdot y z+x z \cdot y) \\
= & 3(x y \cdot z+y z \cdot x+z x \cdot y)-3 x y \cdot z
\end{aligned}
$$

and thus 


$$
J(x, y, z)=3 x y \cdot z \quad \text { where } x, y, z \in A_{a} .
$$

Now $J(x, z, y)=3 x z \cdot y$ and adding this to (7.5) yields $0=x y \cdot z+x z \cdot y$ and with a slight change of notation we have

$$
x y \cdot z=-x \cdot y z \quad \text { where } x, y, z \in A_{a} .
$$

From (7.6) with $z=x$ we obtain

$$
x y \cdot x=0 \text { where } x, y \in A_{a} .
$$

Now let $x, y \in A_{a}, z \in A_{-a}$, then $-a J(x, y, z)=J(x, y, z u)$ and $J(z, y, x u)=a J(z, y, x)=-a J(x, y, z)$. So

$$
\begin{aligned}
-2 a J(x, y, z) & =J(z, y, x u)+J(x, y, z u) \\
& =J(z, y, u) x+J(x, y, u) z=J(x, y, u) z,
\end{aligned}
$$

using (1.7) for the second equality, (2.4) for the third. Thus we have $-2 a J(x, y, z)=J(x, y, u) z=(-3 a x y) z$ using (7.4) and hence

$$
2 J(x, y, z)=3 x y \cdot z \quad \text { where } x, y \in A_{a}, z \in A_{-a} .
$$

This yields $3 x y \cdot z=2(x y \cdot z+y z \cdot x+z x \cdot y)$ or

$$
x y \cdot z=-2(x z \cdot y+x \cdot y z) \text { where } x, y \in A_{a}, z \in A_{-\alpha} .
$$

We now use (7.9) to prove the important identity (7.10). Thus let $w, x, y, z$ be elements of $A_{a}$ and set $v=J(x, y, z), 2 x^{\prime}=y z,-2 y^{\prime}=x z$ and $2 z^{\prime}=x y$. Then

$$
v w=6\left(x^{\prime} w \cdot x+y^{\prime} w \cdot y+z^{\prime} w \cdot z\right) .
$$

To prove this note that $x^{\prime}, y^{\prime}, z^{\prime} \in A_{-a}$ and using (7.9) we have $2 x^{\prime} x \cdot w=$ $x w \cdot x^{\prime}-2 w x^{\prime} \cdot x, 2 y^{\prime} y \cdot w=y w \cdot y^{\prime}-2 w y^{\prime} \cdot y, 2 z^{\prime} z \cdot w=z w \cdot z^{\prime}-2 w z^{\prime} \cdot z$. Adding these equations and multiplying by 2 yield

$$
2 v w=2\left(x w \cdot x^{\prime}+y w \cdot y^{\prime}+z w \cdot z^{\prime}\right)+4\left(x^{\prime} w \cdot x+y^{\prime} w \cdot y+z^{\prime} w \cdot z\right) .
$$

Now using (1.10),

$$
\begin{aligned}
& 2\left(x w \cdot x^{\prime}+y w \cdot y^{\prime}+z w \cdot z^{\prime}\right)=x w \cdot y z+y w \cdot z x+z w \cdot x y \\
& \quad=x(z w \cdot y)+z(w y \cdot x)+w(y x \cdot z)+y(x z \cdot w)+y(x w \cdot z)+x(w z \cdot y) \\
& \quad+w(z y \cdot x)+z(y x \cdot w)+z(y w \cdot x)+y(w x \cdot z)+w(x z \cdot y)+x(z y \cdot w) \\
& \quad=w(y x \cdot z)+w(z y \cdot x)+w(x z \cdot y)+y(x z \cdot w)+z(y x \cdot w)+x(z y \cdot w) \\
& \quad=-w v+y\left(-2 y^{\prime} w\right)+z\left(-2 z^{\prime} w\right)+x\left(-2 x^{\prime} w\right)
\end{aligned}
$$

noting some cancellation to obtain the third equality. Thus $2 v w=$ $v w+2\left(x^{\prime} w \cdot x+y^{\prime} w \cdot y+z^{\prime} w \cdot z\right)+4\left(x^{\prime} w \cdot x+y^{\prime} w \cdot y+z^{\prime} w \cdot z\right)$ and this proves (7.10). 
Since $A$ is simple non-Lie Malcev algebra, we shall use the facts $A^{2}=A$ and $A=J(A, A, A)$ to obtain more identities for $A$. First we have

$$
\begin{aligned}
A_{0} \oplus & A_{\alpha} \oplus A_{-\alpha}=A=J(A, A, A) \\
& \subset J\left(A_{0}, A, A\right)+J\left(A_{\alpha}, A, A\right)+J\left(A_{-\alpha}, A, A\right) \\
& \subset J\left(A_{0}, A_{\alpha}, A_{\alpha}\right)+J\left(A_{0}, A_{-\alpha}, A_{-\alpha}\right)+J\left(A_{\alpha}, A_{\alpha}, A_{\alpha}\right) \\
& +J\left(A_{-\alpha}, A_{-\alpha}, A_{-\alpha}\right)+J\left(A_{\alpha}, A_{\alpha}, A_{-\alpha}\right)+J\left(A_{\alpha}, A_{-\alpha}, A_{-\alpha}\right) \\
& \subset A_{0} \oplus A_{\alpha} \oplus A_{-\alpha}
\end{aligned}
$$

and therefore

$$
\begin{aligned}
A_{0} & =J\left(A_{\alpha}, A_{\alpha}, A_{\alpha}\right)+J\left(A_{-\alpha}, A_{-\alpha}, A_{-\alpha}\right), \\
A_{\alpha} & =J\left(A_{0}, A_{-\alpha}, A_{-\alpha}\right)+J\left(A_{\alpha}, A_{\alpha}, A_{-\alpha}\right), \\
A_{-\alpha} & =J\left(A_{0}, A_{\alpha}, A_{\alpha}\right)+J\left(A_{\alpha}, A_{-\alpha}, A_{-\alpha}\right) .
\end{aligned}
$$

We now use $A=A^{2}$ to obtain

$$
\begin{aligned}
A_{0} \oplus A_{\alpha} \oplus A_{-\alpha} & =A=A^{2} \\
& =A_{0} A_{\alpha}+A_{0} A_{-\alpha}+A_{\alpha}^{2}+A_{\alpha} A_{-\alpha}+A_{-\alpha}^{2}
\end{aligned}
$$

and therefore

$$
\begin{aligned}
A_{0} & =A_{\alpha} A_{-\alpha}, \\
A_{\alpha} & =A_{0} A_{\alpha}+A_{-\alpha}^{2}, \\
A_{-\alpha} & =A_{0} A_{-a}+A_{\alpha}^{2} .
\end{aligned}
$$

Since $A_{0}=u F$ we have $A_{0} A_{a}=A_{a}(\alpha= \pm \alpha)$. Also

$$
\begin{aligned}
J\left(A_{0}, A_{-a}, A_{-a}\right) \subset A_{a}=A_{0} A_{a} \\
\quad \subset A_{0} J\left(A_{0}, A_{-a}, A_{-a}\right)+A_{0} J\left(A_{a}, A_{a}, A_{-a}\right) \\
\quad \subset J\left(A_{0}, A_{0}, A_{-a}^{2}\right)+J\left(A_{0}, A_{-a}, A_{-a} A_{0}\right)+J\left(A_{0}, A_{-a}, A_{0} A_{-a}\right) \\
\quad+J\left(A_{0}, A_{a}, A_{a} A_{-a}\right)+J\left(A_{0}, A_{a}, A_{-a} A_{a}\right)+J\left(A_{0}, A_{-a}, A_{a}^{2}\right) \\
\quad \subset J\left(A_{0}, A_{-a}, A_{-a}\right),
\end{aligned}
$$

obtaining the second inclusion from $A_{a}=J\left(A_{0}, A_{-a}, A_{-a}\right)+J\left(A_{a}, A_{a}, A_{-a}\right)$ and the third inclusion from (1.8). Thus we have

$$
A_{a}=J\left(A_{0}, A_{-a}, A_{-a}\right), \quad a \neq 0 .
$$

From this and remembering $A_{0}=u F$ we obtain

$$
A_{a}=A_{-a} A_{-a}, \quad a \neq 0 .
$$

For $A_{-a} A_{-a} \subset A_{a}=J\left(A_{0}, A_{-a}, A_{-a}\right) \subset A_{-a} A_{-a}$. Also

$$
A_{0}=J\left(A_{a}, A_{a}, A_{a}\right), \quad a= \pm \alpha \text {. }
$$

For 


$$
\begin{aligned}
& J\left(A_{a}, A_{a}, A_{a}\right) \subset A_{0}=A_{a} A_{-a} \\
& \quad=A_{a} J\left(A_{0}, A_{a}, A_{a}\right) \\
& \quad \subset J\left(A_{a}, A_{0}, A_{a}^{2}\right)+J\left(A_{a}, A_{a}, A_{a} A_{0}\right)+J\left(A_{a}, A_{a}, A_{0} A_{a}\right) \\
& \quad \subset J\left(A_{a}, A_{a}, A_{a}\right) .
\end{aligned}
$$

We summarize these identities in

Proposition 7.11. Let $A=A_{0} \oplus A_{\alpha} \oplus A_{-\alpha}$ be the usual simple nonLie Malcev algebra, then we have for $a= \pm \alpha$,

$$
A_{a}=A_{0} A_{a}=A_{-a} A_{-a}
$$

and

$$
A_{0}=A_{a} A_{-a}=J\left(A_{a}, A_{a}, A_{a}\right)
$$

Theorem 7.12. Let $A=A_{0} \oplus A_{\alpha} \oplus A_{-\alpha}$ be the usual simple nonLie Malcev algebra, then $A$ is isomorphic to the simple seven dimensional Malcev algebra $A^{*}$ discussed in the introduction.

Proof. Since $u F=A_{0}=A_{\alpha} A_{-\alpha}=A_{\alpha} \cdot A_{\alpha} A_{\alpha}$, there exists $x, y, z \in A_{\alpha}$ such that $x \cdot y z=2 u$. Define $2 x^{\prime}=y z,-2 y^{\prime}=x z$ and $2 z^{\prime}=x y$ and form the subspace $B$ generated by $\left\{u, x, y, z, x^{\prime}, y^{\prime}, z^{\prime}\right\}$. First the $x, y$ and $z$ are linearly independent over $F$. For if $a x+b y+c z=0$ with $a, b, c \in F$ and, for example, $a \neq 0$, then write $x=b^{\prime} y+c^{\prime} z$ and therefore using (7.7) $2 u=x \cdot y z=b^{\prime} y \cdot y z+c^{\prime} z \cdot y z=0$, a contradiction. Similarly noting $u=x x^{\prime}$ and assuming a relation of the type $x^{\prime}=$ $b^{\prime} y^{\prime}+c^{\prime} z^{\prime}$ and using the definitions of $x^{\prime}, y^{\prime}$ and $z^{\prime}$ we see that the $x^{\prime}, y^{\prime}$ and $z^{\prime}$ are also linearly independent. Since $A=A_{0} \oplus A_{\alpha} \oplus A_{-\alpha}$, $\left\{u, x, y, z, x^{\prime}, y^{\prime}, z^{\prime}\right\}$ is a linearly independent set of vectors over $F$. Using identities (1.2), (7.6) and (7.7) we obtain the following multiplication table for $B$.

\begin{tabular}{c|ccccccc} 
& $u$ & $x$ & $y$ & $z$ & $x^{\prime}$ & $y^{\prime}$ & $z^{\prime}$ \\
\hline$u$ & 0 & $-\alpha x$ & $-\alpha y$ & $-\alpha z$ & $\alpha x^{\prime}$ & $\alpha y^{\prime}$ & $\alpha z^{\prime}$ \\
$x$ & $\alpha x$ & 0 & $2 z^{\prime}$ & $-2 y^{\prime}$ & $u$ & 0 & 0 \\
$y$ & $\alpha y$ & $-2 z^{\prime}$ & 0 & $2 x^{\prime}$ & 0 & $u$ & 0 \\
$z$ & $\alpha z$ & $2 y^{\prime}$ & $-2 x^{\prime}$ & 0 & 0 & 0 & $u$ \\
$x^{\prime}$ & $-\alpha x^{\prime}$ & $-u$ & 0 & 0 & 0 & $\alpha z$ & $-\alpha y$ \\
$y^{\prime}$ & $-\alpha y^{\prime}$ & 0 & $-u$ & 0 & $-\alpha z$ & 0 & $\alpha x$ \\
$z^{\prime}$ & $-\alpha z^{\prime}$ & 0 & 0 & $-u$ & $\alpha y$ & $-\alpha x$ & 0
\end{tabular}

By the remarks at the beginning of this section we can choose $\alpha=-2$ 
and consequently obtain that $B$ is isomorphic to $A^{*}$. It remains to show the dimension of $A$ over $F$ is seven. For this it suffices to show dimension $A_{\alpha}=3$, since dimension $A_{\alpha}=\operatorname{dimension} A_{-\alpha}$. Let $0 \neq w \in A_{\alpha}$, then by $(7.5)$

$$
6 u=3 x \cdot y z=-J(x, y, z)
$$

and therefore by (7.10),

$$
6 \alpha w=6 w u=x_{0} x+y_{0} y+z_{0} z
$$

where $x_{0}, y_{0}, z_{0} \in A_{0}=u F$. But by the action of $u$ on $x, y$ and $z$ we have $6 \alpha w=a_{0} x+b_{0} y+c_{0} z$ where $a_{0}, b_{0}, c_{0} \in F$. Thus the dimension of $A_{\alpha}$ is three.

\section{BIBLIOGRAPHY}

1. A. A. Albert, On simple alternative rings, Canadian J. Math., 4 (1952), 129-135.

2. N. Jacobson, Lectures in abstract algebra, Vol. 2, D. Van Norstrand.

3. A. Sagle, Malcev algebras, Trans. Amer. Math. Soc., 101 (1961), 426-458.

The University of Chicago and

SRNACUSE UNIVERSITY 


\title{
PACIFIC JOURNAL OF MATHEMATICS
}

\author{
EDITORS
}

RalPh S. Phillips

Stanford University

Stanford, California

M. G. Arsove

University of Washington

Seattle 5 , Washington
A. L. Whiteman

University of Southern California Los Angeles 7, California

LOWell J. PAIGE

Unıversity of California

Los Angeles 24, California

\section{ASSOCIATE EDITORS}
E. F. BECKENBACH
D. DERRY
H. L. ROYDEN
E. G. STRAUS
T. M. CHERRY
M. OHTSUKA
E. SPANIER
F. WOLF

\section{SUPPORTING INSTITUTIONS}

\author{
UNIVERSITY OF BRITISH COLUMBIA \\ CALIFORNIA INSTITUTE OF TECHNOLOGY \\ UNIVERSITY OF CALIFORNIA \\ MONTANA STATE UNIVERSITY \\ UNIVERSITY OF NEVADA \\ NEW MEXICO STATE UNIVERSITY \\ OREGON STATE UNIVERSITY \\ UNIVERSITY OF OREGON \\ OSAKA UNIVERSITY \\ UNIVERSITY OF SOUTHERN CALIFORNIA
}

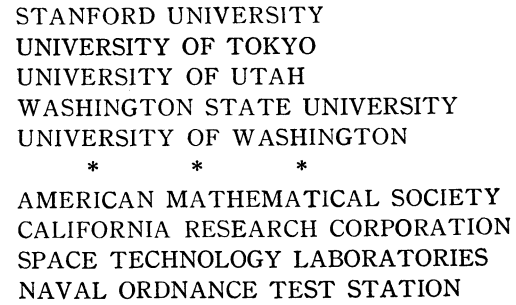

Mathematical papers intended for publication in the Pacific Journal of Mathematıcs should be typewritten (double spaced), and the author should keep a complete copy. Manuscripts may be sent to any one of the four editors. All other communications to the editors should be addressed to the managing editor, L. J. Paige at the University of California, Los Angeles 24, California.

50 reprints per author of each article are furnıshed free of charge; additional copies may be obtained at cost in multiples of 50 .

The Pacific Journal of Mathematics is published quarterly, in March, June, September, and December. Effective with Volume 13 the price per volume (4 numbers) is $\$ 18.00$; single issues, $\$ 5.00$. Special price for current issues to individual faculty members of supporting institutions and to individual members of the American Mathematical Society: $\$ 8.00$ per volume; single issues $\$ 2.50$. Back numbers are available.

Subscriptions, orders for back numbers, and changes of address should be sent to Pacific Journal of Mathematics, 103 Highland Boulevard, Berkeley 8, California.

Printed at Kokusai Bunken Insatsusha (International Academic Printing Co., Ltd.), No. 6 , 2-chome, Fujimi-cho, Chiyoda-ku, Tokyo, Japan.

PUBLISHED BY PACIFIC JOURNAL OF MATHEMATICS, A NON-PROFIT CORPORATION

The Supporting Institutions listed above contribute to the cost of publication of this Journal, but they are not owners or publishers and have no responsibility for its content or policies. 


\section{Pacific Journal of Mathematics}

\section{Vol. 12, No. $3 \quad$ March, 1962}

Alfred Aeppli, Some exact sequences in cohomology theory for Kähler

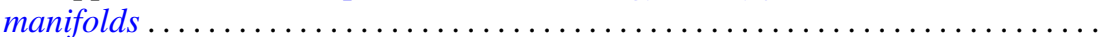

Paul Richard Beesack, On the Green's function of an N-point boundary value

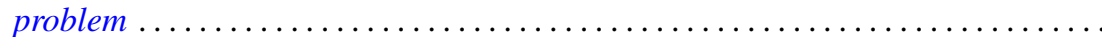

James Robert Boen, On p-automorphic p-groups....

James Robert Boen, Oscar S. Rothaus and John Griggs Thompson, Further results

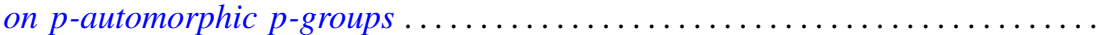

James Henry Bramble and Lawrence Edward Payne, Bounds in the Neumann problem for second order uniformly elliptic operators ..................

Chen Chung Chang and H. Jerome (Howard) Keisler, Applications of ultraproducts of pairs of cardinals to the theory of models .........................

Stephen Urban Chase, On direct sums and products of modules ................

Paul Civin, Annihilators in the second conjugate algebra of a group algebra .......

J. H. Curtiss, Polynomial interpolation in points equidistributed on the unit

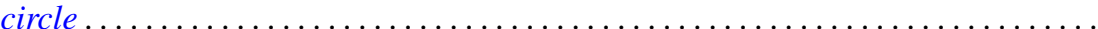

Marion K. Fort, Jr., Homogeneity of infinite products of manifolds with

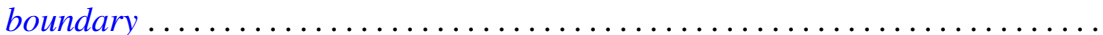

James G. Glimm, Families of induced representations . . . . . . . . . . . . . . .

Daniel E. Gorenstein, Reuben Sandler and William H. Mills, On almost-commuting

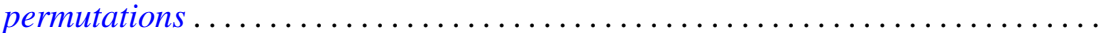

Vincent C. Harris and M. V. Subba Rao, Congruence properties of $\sigma_{r}(N) \ldots \ldots \ldots$

Harry Hochstadt, Fourier series with linearly dependent coefficients . . . . . . . . . . 925

Kenneth Myron Hoffman and John Wermer, A characterization of $C(X)$. .

Robert Weldon Hunt, The behavior of solutions of ordinary, self-adjoint differential equations of arbitrary even order...

Edward Takashi Kobayashi, A remark on the Nijenhuis tensor

David London, On the zeros of the solutions of $w^{\prime \prime}(z)+p(z) w(z)=0$

Gerald R. Mac Lane and Frank Beall Ryan, On the radial limits of Blaschke products...

T. M. MacRobert, Evaluation of an E-function when three of its upper parameters

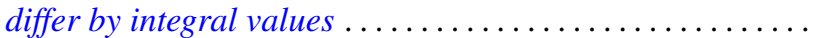

Robert W. McKelvey, The spectra of minimal self-adjoint extensions of a symmetric operator

Adegoke Olubummo, Operators of finite rank in a reflexive Banach space. .

David Alexander Pope, On the approximation of function spaces in the calculus of variations

Bernard W. Roos and Ward C. Sangren, Three spectral theorems for a pair of singular first-order differential equations...............

Arthur Argyle Sagle, Simple Malcev algebras over fields of characteristic zero .

S. C. Tang, Some theorems on the ratio of empirical distribution to the theoretical distribution

Robert Charles Thompson, Normal matrices and the normal basis in abelian number fields.

Howard Gregory Tucker, Absolute continuity of infinitely divisible distributions ... 